\title{
Experimental infection model with the bivalvulid Enteromyxum leei (Myxidiidae) in the sharpsnout seabream, Diplodus puntazzo (Sparidae), and evaluation of the antiparasitic efficacy of a functional diet
}

\author{
Morgane A. Henry ${ }^{1}$, Fotini Kokou ${ }^{1,2}$, Oswaldo Palenzuela ${ }^{3}$, George Pyrenis $^{1}$ and George Rigos ${ }^{1}$ \\ ${ }^{1}$ Institute of Marine Biology, Biotechnology and Aquaculture, Hellenic Centre for Marine Research, Anavissos, Attiki, Greece; \\ ${ }^{2}$ Aquaculture and Fisheries Group, Wageningen Institute of Animal Sciences, Wageningen University, Wageningen, The Netherlands; \\ ${ }^{3}$ Institute of Aquaculture Torre de la Sal (IATS-CSIC), Castellón, Spain.
}

\begin{abstract}
An infection model for sharpsnout seabream Diplodus puntazzo (Walbaum) challenged with the myxosporean Enteromyxum leei (Diamant, Lom et Dyková, 1994), resembling the natural infection conditions, was used to evaluate the antiparasitic efficacy of a functional diet. Fish of an average weight of $12.5 \pm 1.2 \mathrm{~g}$ were delivered either a functional (included as feed supplement at $0.3 \%$ levels) or a control extruded diet. After four weeks of administration of the experimental diets, fish were challenged with the parasites (cohabitation with infected donors; donor: recipient ratio $1: 1$ ). The experiment was terminated four weeks after the start of the challenge. At the end of the experiment, growth and feeding (specific growth rate and feed efficiency), as well as immunological parameters (respiratory burst activity, antibacterial activities, hemoglobin concentration, anti-protease activity and ceruloplasmin activity) were measured along with cumulative mortality and total parasitic count in the gut. No significant difference was evident with regard to growth and feeding performance, mortality, gut parasitic load or immunological parameters as the parasitical challenge significantly affected both the performance of the control and functional diet fed fish. However, there was a less prominent impact on antibacterial, anti-protease and ceruloplasmin activity in fish fed with the functional diet. Overall, the present study validated the experimental cohabitation infection model and evaluated the efficacy of a functional ingredient as an antiparasitic agent, showing some potential effects on the fish immune response.
\end{abstract}

Keywords: Cohabitation parasitical challenge, Myxosporea, immune response, hemoglobin, inflammation, gut parasite, parasite intensity, parasite prevalence, qPCR.

The farming of sharpsnout seabream, Diplodus puntazzo (Walbaum), was considered quite promising in its first attempts in the 1990s and early 2000s because of a fast growth and a high market value (Favaloro et al. 2002, Favaloro and Mazzola 2003, Hernández et al. 2008). Despite the successful early stage rearing of this species, it has been abandoned in most aquaculture enterprises in the Mediterranean due to enteromyxosis, a disease caused by the myxozoan parasite Enteromyxum leei (Diamant, Lom et Dyková, 1994) (syn. Myxidium leei) (Diamant et al. 1994, Le Breton and Marques 1995, Diamant 1997, Rigos et al. 1999, Athanasopoulou et al. 2001, Golomazou et al. 2006).

This parasite is responsible of serious epizootic outbreaks with severe catarrhal enteritis, which usually causes high fish mortality under extreme cachectic conditions few weeks after introduction to the on-growing net cages (Al-
varez-Pellitero et al. 2008, Sitjà-Bobadilla and Palenzuela 2011). The disease courses faster and with higher mortality when water temperatures are high (Palenzuela 2006, Alvarez-Pellitero et al. 2008, Sánchez-García et al. 2014).

Enteromyxum leei, as most species of Enteromyxum Palenzuela, Redondo et Álvarez-Pellitero, 2002, is directly transmitted by fish-to-fish contact favouring the propagation of the parasite in intense farming conditions. Fish can be infected through exposure to a contaminated water, by cohabitation with infected fish or by ingestion of pre-sporogonic developmental stages (Diamant 1997). Specifically, in enteromyxosis, trophozoites of E. leei are responsible for the parasite proliferation in the host, by invading new intestinal areas but also for the transmission to healthy fish when they are released from faecal products of infected individuals (Cuadrado et al. 2010). 


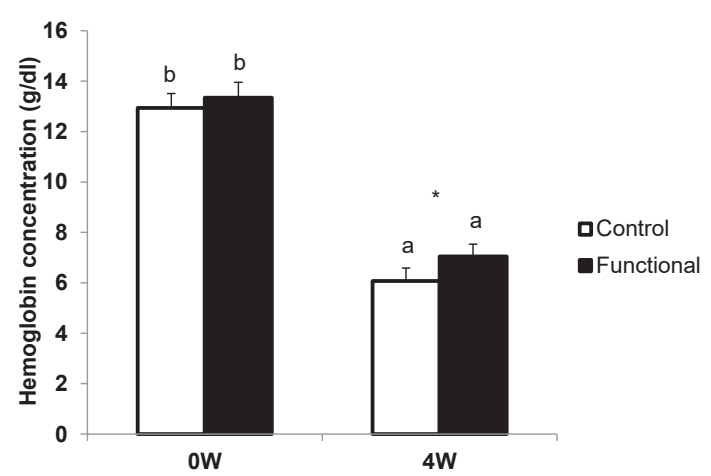

Fig. 1. Hemoglobin concentration ( $\mathrm{g} / \mathrm{dl}$ blood) in the fresh blood of fish fed control or functional diets for 4 weeks before $(0 \mathrm{~W})$ and 4 weeks $(4 \mathrm{~W})$ after the parasitical challenge by cohabitation. Bars represent mean \pm S.E. Different letters represent a significant difference between treatments (One-Way ANOVA, $\mathrm{P}=3.5 \times 10^{-16}$ ). The asterisk shows a significant difference between the 2 sampling times (General Linear Method, $\mathrm{P}=1.9 \times 10^{-7}$ ). Sample size, $\mathrm{n}=15$ at $0 \mathrm{~W} ; \mathrm{n}=42$ at $4 \mathrm{~W}$.

There are currently no prescription medicines effective against this parasite and most attempts to control it in the field and/or in experimental trials have failed (Sitjà-Bobadilla et al. 2008, Sánchez-García et al. 2014). However, empirical evidence in the farms and recent laboratory-controlled experiments have shown promising results in the control of enteromyxosis in gilthead seabream, Sparus aurata (Linnaeus) using some functional feed additives (Palenzuela et al. 2017, Piazzon et al. 2017, Picard-Sánchez et al. 2019).

Functional feed additives are currently the focus for disease prevention and control, with the aim to reduce the antimicrobial control agents as well as to reduce the environmental risks (Defoirdt et al. 2011). These include a wide range of ingredients, such as probiotics, phytobiotics, yeast extracts or organic acids, which can act in multiple ways that promote the immune system and may reduce the parasitic infections. Natural compounds with anti-parasitic activity can directly affect gut parasites or indirectly affect ectoparasites through their effects on blood or the composition and quantity of the mucus (Coutteau 2016).

To our knowledge, scarce studies exist so far regarding the effects of functional feed additives on the sharpsnout seabream resistance to enteromyxosis. Therefore, the aim of the present work was (i) to validate an experimental infection model with E. leei in sharpsnout seabream, and (ii) to evaluate the antiparasitic effects of a natural health promoting feed additive by measuring growth, survival, immunological parameters and parasitic load.

\section{MATERIALS AND METHODS}

The research complied with the commonly-accepted '3Rs' and the ARRIVE (Animal Research: Reporting of in vivo Experiments) guidelines. All the procedures involving fish were performed according to the EU guidelines on the protection of animals used for scientific purposes (Directive 2010/63/EU). Five hundred parasite-free sharpsnout seabream juveniles $(\sim 2 \mathrm{~g})$ were transferred from the hatchery of a commercial farm (Selonda, east

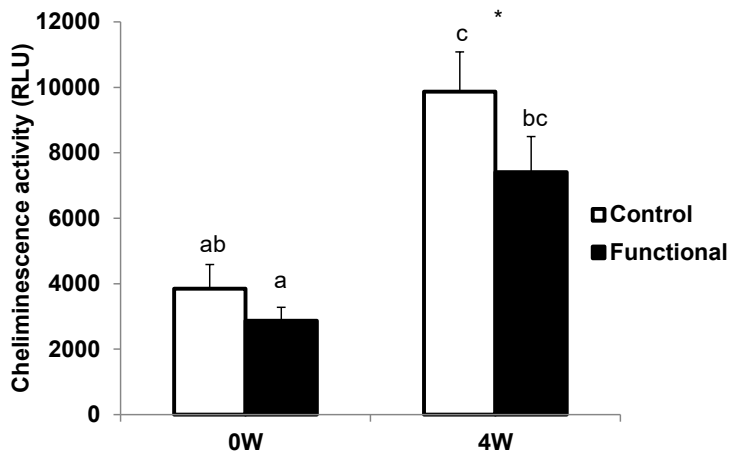

Fig. 2. Chemiluminescence activity (Relative Luminescent Units) in the whole blood of fish fed control or functional diets for 4 weeks before ( $0 \mathrm{~W})$ and 4 weeks ( $4 \mathrm{~W}$ ) after the parasitical challenge by cohabitation. Bars represent mean \pm S.E. Different letters represent a significant difference between treatments of the $\log _{10}$-transformed data (One-Way ANOVA, $\mathrm{P}=0.001$ ). The asterisk shows a significant difference of the transform data between the two sampling times (General Linear Method, $\mathrm{P}=0.0003$ ). Sample size, $\mathrm{n}=15$ at $0 \mathrm{~W} ; \mathrm{n}=42$ at $4 \mathrm{~W}$.

Peloponnesus) to the indoor facilities of the Hellenic Centre for Marine Research (HCMR) in Athens, Greece. Maintenance of sharpsnout seabream in land tanks ensures the avoidance of infection with Enteromyxum leei, which is inevitably initiated when fish enter the sea cages. Upon arrival, 30 fish were examined (microscopic observation of fresh smears of the intestine) to ascertain the absence of the parasite. Fish were maintained in 2501 fibreglass tanks supplied with open-flow water along with aeration and temperature ranging from $18-20^{\circ} \mathrm{C}$. The animals were given a commercial diet until they reached a size of $12.5 \pm 1.2 \mathrm{~g}$, and were then transferred to a large $\left(50 \mathrm{~m}^{3}\right)$ inland cement tank and equally distributed (300 fish) in six identical net cages ( $1 \mathrm{~m}^{3 ;} 50$ fish/cage). Three of these cages served as the control group and the three remaining cages as the functional diet group. In this environment, experimental fish were delivered either control or functional extruded diets prepared in the facilities. The functional diet included Sanacore ${ }^{\circledR}$ GM, a commercial natural health promoting feed additive (Adisseo, Dendermonde, Belgium) at $0.3 \%$ inclusion levels. The composition of the diets is provided in Table 1 .

Throughout the trial, feed consumption was daily recorded. Four weeks after the initiation of the dietary trial, a challenge trial was performed for an additional four-week-period by cohabitation with diseased fish. More specifically, 500 sharpsnout seabream (mean weight $30 \mathrm{~g}$ ) from a naturally-occurring outbreak of E. leei at a commercial farm (Selonda, east Peloponnesus), were transferred to HCMR facilities. To ensure that the fish were carrying the parasites, fresh intestinal smears of 30 fish were examined microscopically.

Following confirmation of the pathogen presence, 300 donor/ diseased fish were introduced into the cement tank harbouring the caged recipient/healthy fish (giving a $1: 1$ donor : recipient ratio) and were free to roam outside the net cages without interfering with the feeding of the recipient fish. Donor fish remained unfed until the termination of the trial. Fish tanks were continuously supplied with aerated flow-through sea water. The experiment was terminated four weeks after the start of the challenge and the temperature during the trial ranged from 24 to $27^{\circ} \mathrm{C}$. Fish mortalities were monitored daily. 

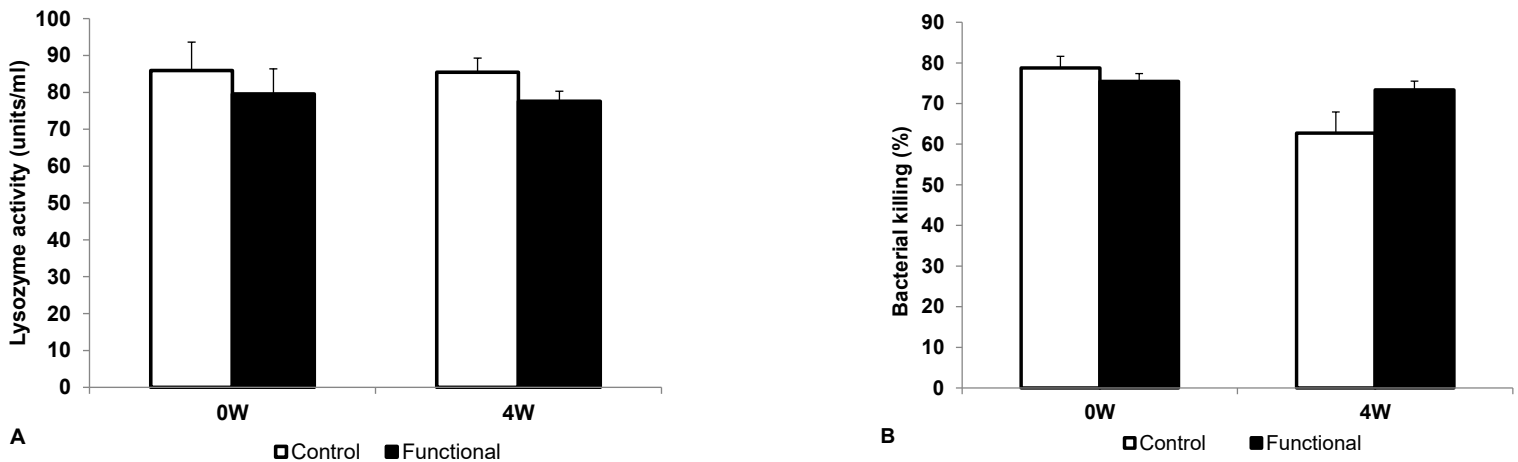

Fig. 3. Serum antibacterial activity (A, anti-Gram positive; B, anti-Gram-negative bacteria) of fish fed control or functional diets for 4 weeks before $(0 \mathrm{~W})$ and 4 weeks $(4 \mathrm{~W})$ after the parasitical challenge by cohabitation. Bars represent mean \pm S.E. There was no significant difference between treatments (One-Way ANOVA for $\log _{10}$ anti- $\mathrm{G}^{+}$, Welch for anti $\mathrm{G}-$ bacteria, $\mathrm{P}>0.05$ ). Sample size, $\mathrm{n}=15$ at $0 \mathrm{~W} ; \mathrm{n}=42$ at $4 \mathrm{~W}$.

Donors and recipients fish were sampled twice, immediately prior to the beginning of the challenge and at the termination of the experiment, four weeks later. Samples were taken in order to measure immunological parameters and for the diagnosis and quantification of E. leei small subunit ribosomal RNA gene (SSU rRNA) by qPCR. Prior to sampling, fish were anaesthetised with clove oil (50 ppm) and after blood sampling via the caudal vein fish were killed by overdose of anaesthetic. An aliquot of blood was heparinised (500 units $/ \mathrm{ml}$ ) and it was used for the determination of the hemoglobin concentration and the respiratory burst activity, while the remaining non-heparinised blood was left to clot overnight at $4{ }^{\circ} \mathrm{C}$, centrifuged, and the supernatant sera were frozen at $-80^{\circ} \mathrm{C}$ until used for the determination of lysozyme, complement, anti-protease and ceruloplasmin activity.

In addition, fish were necropsied and the whole intestines were removed from the pyloric area to the rectum. They were preserved in $80 \%$ ethanol for parasite diagnosis and quantification. Before the challenge, five fish were sampled from each cage (15 per group), while 14 fish were sampled at the end of the challenge (42 per group) for immunological measurements. For parasitological determination, samples were taken from donor fish (30 at the beginning and 20 at the end of the trial) and from 15 fish per cage (45 per group) at the termination of the experiment. When fish could be collected rapidly after death, intestine samples were also collected from dead fish. Gut samples were stored in ethanol for qPCR analysis.

- Feed Conversion Rate, FCR = FI / WG

- Specific Growth Rate, SGR $=100 \times[\ln (\mathrm{W} 1)-\ln (\mathrm{W} 0)] /$ rearing days;

- Feed Efficiency, FE $=(\mathrm{W} 1-\mathrm{W} 0) \times 100 / \mathrm{FI}($ where FI is the feed intake, WG is the wet weight gain, W0 and W1 are the initial and the final fish mean weights respectively, all expressed in grams).

Hemoglobin concentration was measured as previously described for gilthead seabream (Rigos et al. 2010) and the whole blood respiratory burst activity was determined by assessing the chemiluminescence produced by circulating cells in the diluted blood in contact with yeast cell walls (zymosan A), as for European sea bass, Dicentrarchus labrax (Linnaeus) (Henry et al. 2009). The serum antibacterial activities were determined against a Gram-positive bacterium, Micrococcus luteus (i.e., lysozyme activity in units/ml) and against a Gram negative bacterium, Escherichia coli (percentage of bacterial killing associated mainly to complement activity). The procedure was adapted from the methods previously described for gilthead seabream (Kokou et al. 2012, Henry et al. 2015). The anti-protease and ceruloplasmin activities were determined as previously described for shi drum Umbrina cirrosa (Linnaeus) (Henry and Fountoulaki 2014).

For the parasitology, gene copies of the E. leei SSU rRNA were estimated by qPCR as previously described (Piazzon et al. 2017). Ethanol-fixed intestines from sharpsnout seabream were briefly hydrated and weighed individually. They were homogenised in a laboratory blender (Next Advance, NY, USA) and DNA was extracted from a $200 \mu \mathrm{l}$ aliquot of the homogenate from each fish using an EpMotion 5075 automated robotic system (Eppendorf, Hamburg, Germany) and commercial silica-based kits (Macherey-Nagel, Düren, Germany).

Numbers were interpolated from the cycle thresholds $(\mathrm{Ct})$ of the samples using standard curves generated with known numbers of a plasmid containing the target gene (covering 6-7 orders of magnitude), run in the same plates for each assay. Usually two dilutions of each DNA sample were run. Samples with $\mathrm{Ct}$ $<38$ were considered positive. The total number of parasite SSU rRNA genes present in each fish was estimated from these values and the amount of tissue in the entire homogenate sample. For simplicity, this value was used as a proxy to the number of parasite cells (i.e., assuming a constant SSU rRNA copy number per parasite).

For the statistical analysis, all values are presented as means \pm standard errors of the means. SPSS 13.0 software was used for all statistical analysis. Normal distribution and homogeneity of variance were checked using Kolmogorov-Smirnov and Levene tests, respectively. When normality was not obtained (i.e., for chemiluminescence, lysozyme and ceruloplasmin), variables were transformed using logarithm base $10(\log )$. General Linear Method (GLM) was used to determine differences between diets, time or diet $\times$ time. Immune parameters were also checked for difference between experimental diets and time (before and after the parasitical challenge) by one-Way ANOVA or Welch and Tukey's or Games-Howel $t$-tests when appropriate. Concerning the DNA data, Mann-Whitney or $t$-test were used. Statistical significance was considered when $\mathrm{P}<0.05$. 


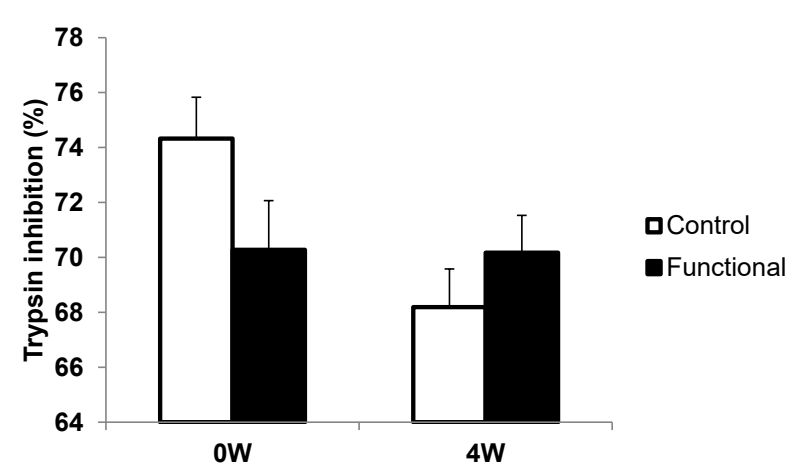

Fig. 4. Serum anti-protease activity of fish fed control or functional diets for 4 weeks before $(0 \mathrm{~W})$ and 4 weeks $(4 \mathrm{~W})$ after the parasitical challenge by cohabitation. Bars represent mean \pm S.E. There was no significant difference between treatments (GLM and One-Way ANOVA, $\mathrm{P}>0.05)$. Sample size, $\mathrm{n}=15$ at $0 \mathrm{~W}$; $\mathrm{n}=42$ at $4 \mathrm{~W}$.

\section{RESULTS}

\section{Growth and survival}

Growth of sharpsnout seabream was not significantly different between control and functional feed fed group ( $\mathrm{P}>0.05$ : Table 2). However, the overall performance was greatly affected by infection with Enteromyxum leei as reflected by the calculated growth parameters, with minor differences between the two groups (Table 2). Specific growth rate ranged between 0.55 and $0.58 \%$, while feed efficiency was low in both groups, with FE ranging from 33 to $35 \%$ and FCR ranging from 2.9 to 3.0.

No mortality of the recipient sharpsnout seabream was observed during the pre-growth adaptation phase or during the prophylaxis feeding trial before the introduction of the donor-infected fish. Approximately 10 days after the start of the cohabitation challenge, the first mortalities emerged in both groups indicating the success of the cohabitation challenge, which was ascertained through the observation of parasites in bile and gut smears. Eventually, the cumulative mortality after challenge reached values as high as 22 and $27 \%$ in the control and functional feed fed group, respectively (Table 2 ). The differences in fish survival between functional and control groups were not significant $(\mathrm{P}>0.05)$.

\section{Immunological parameters}

Hemoglobin concentration was not affected by the diet (GLM, P >0.05), but was significantly reduced by the parasite challenge (GLM, P $=1.9 \times 10^{-7}$; Fig. 1 ). Similarly, whole blood respiratory burst activity was affected by the parasitical challenge $(\mathrm{P}=0.0003)$ but not by the diet (P>0.05; Fig. 2).

The antibacterial activities against a Gram-positive bacterium, Micrococcus luteus (i.e., the lysozyme activity) and against a Gram-negative bacterium, E. coli, (i.e., complement activity), are presented in Fig 3B. Insignificant differences were obtained between treatments. However, a tendency for a decrease in antibacterial activity was ob-

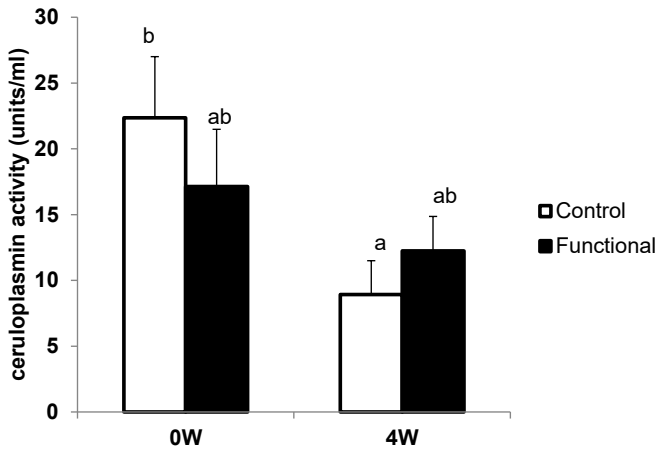

Fig. 5. Serum ceruloplasmin activity of fish fed control or functional diets for 4 weeks before $(0 \mathrm{~W})$ and 4 weeks (4W) after the parasitical challenge by cohabitation. Bars represent mean \pm S.E. There was no significant difference of the transform data $\left(\log _{10}\right)$ between treatments (General Linear Method and One-Way ANOVA, $\mathrm{P}>0.05)$. Sample size, $\mathrm{n}=15$ at $0 \mathrm{~W} ; \mathrm{n}=42$ at $4 \mathrm{~W}$.

served in control fish after the parasite challenge, whereas the functional group maintained similar values despite the challenge (13\% decrease in control vs. $2 \%$ decrease in the functional group; Fig. 3B). This tendency for a stabilising role of the functional diet against parasitical challenge-induced reduction of immune parameters was also observed concerning the anti-protease activity ( $8 \%$ decrease in control vs. $0.2 \%$ decrease in the functional group; Fig. 4) and the ceruloplasmin activity ( $60 \%$ decrease in control vs. 30 $\%$ decrease in the functional group; Fig. 5) of the fish sera.

\section{Parasitological examination}

Out of the 90 experimental fish examined, 69 were positive for E. leei by qPCR (77\% prevalence). Of these, 35 belonged to the control group ( $77 \%$ prevalence) and 34 to the functional feed group (76\%). No differences were found in the prevalence of infection between groups or among tanks within each group. The intensity of infection in experimental fish was quite variable, encompassing $\mathrm{Ct}$ values from 16 to 30 . These correspond to a four-fold increase in parasite SSU rRNA gene copies per infected fish $\left(3.7 \times 10^{5}\right.$ to 3.9 $\times 10^{9}$ copies). Using transformed data, the mean intensity of infection was $17 \pm 0.5$ (corresponding to $1.57 \times 10^{7}$ copies-fish $\left.{ }^{-1}\right)$. No significant difference in the mean intensity was found between control $(17 \pm 0.6)$ and functional feed fed sharpsnout seabream $(17 \pm 0.9)$ (t-test; $p>0.05)$. The range of data distribution was wider in functional feed fed fish, which yielded the lowest and the highest values. The median was also slightly lower in functional feed fed fish (16.06) than in control fish (16.33) but these differences were not significant (Mann-Whitney $\mathrm{p}=1$ ) (Fig. 6).

Parasite prevalence in donor fish was lower than in experimental fish (62\%), and this value decreased from $67 \%$ to $55 \%$ during the trial. In addition, donors presented lower intensity values $(14 \pm 1)$ than experimental fish and this difference was statistically significant (ANOVA $p<0.001$ ). The mean intensity of infection in donor fish also decreased along the trial, from $14.3 \pm 1.3$ to $13.0 \pm 2.1$. These differences, however, were not statistically significant $(t$-test $\mathrm{p}>0.05)$. 
Intensity copies/fish (Ln transformed data)

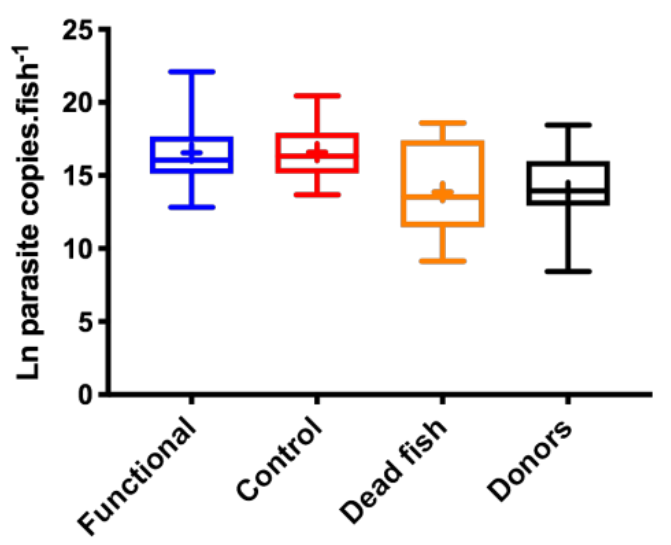

\section{Intensity copies/fish (Ln transformed data)}

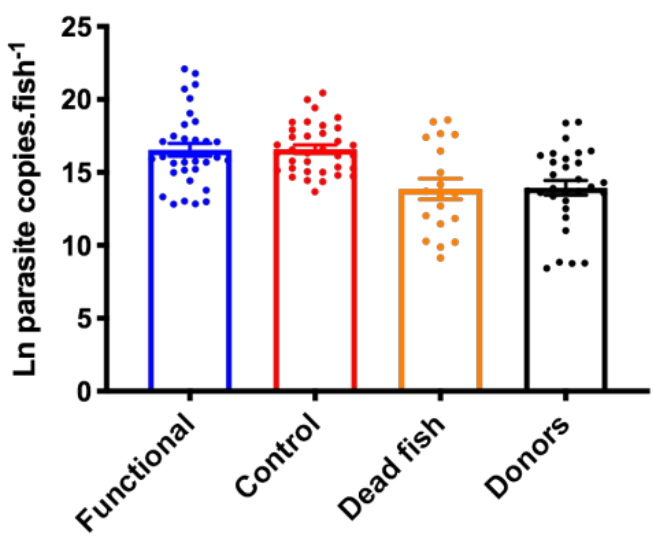

Fig. 6. Parasitic infection intensity (parasite SSU rDNA copies fish ${ }^{-1}$ ) in the experimental groups, recipient fish fed either with functional feed or control diet, collected dead fish and donor fish. Data are ln transformed to achieve normal distribution of the data. Left: Box and whiskers plot: Boxes extend from 25 to $75 \%$ percentiles and the lines within the boxes represent the median of the group. A "+" symbol marks the mean value of the group. Right: bars represent mean $\pm \mathrm{SE}$ for each group. Only infected fish $(\mathrm{Ct}<38)$ are considered and all individual fish data are plotted. $\mathrm{n}=19-45$.

Parasite prevalence in dead fish was $63 \%$ and mean intensity of infection was $14 \pm 1.5$. While these values were similar to those of donor fish, they were significantly lower than in experimental fish at the end of the trial. The fact that not all dead fish were found to be infected, and their relatively low intensity values, seem to suggest that some of these post-mortem samples retrieved from carcasses were degraded and the values would not accurately reflect the status of these fish.

\section{DISCUSSION}

In the present work, a host-parasite model for experimental infection of sharpsnout seabream with Enteromyxum leei was evaluated. Transmission of E. leei from infected donors to healthy recipient sharpsnout seabream by cohabitation was achieved. Mortality of the infected recipient fish, as ascertained through the presence of E. leei in the digestive system, started within the second week post challenge. Indeed, the model successfully resembled the progression of the disease observed in the field and the disease pattern seen in gilthead seabream, although faster and in a more intense manner (Sitjà-Bobadilla et al. 2008). Moreover, the high prevalence and intensity of the parasitic infection and the observed disease symptoms reflected the characteristics of the pathology found in the production units.

A previous successful cohabitation challenge model using E. leei in $10-50 \mathrm{~g}$ sharpsnout seabream at $18-21^{\circ} \mathrm{C}$ failed to cause fish mortalities (Golomazou et al. 2006, Muñoz et al. 2007, Alvarez-Pellitero et al. 2008). The high water temperature in the present trial $\left(24-27^{\circ} \mathrm{C}\right)$ was most probably responsible for the severity of the infection, causing high mortalities both in the control and the functional diet group. However, the impaired growth and immunological parameters (reduced hemoglobin concentration and increased respiratory burst activity) due to enteromyxosis are in agreement with a previous study (Golomazou et al. 2006).
Concerning the immunological parameters, the parasitical challenge significantly affected fish fed both the control and the functional diet. This was obvious through the reduced hemoglobin concentration and the increased blood respiratory burst activity caused by the parasitical challenge. This pattern was reported before (Alvarez-Pellitero et al. 2008) 20 and 27 days after the start of the challenge concerning the reduction of hemoglobin, whereas the chemiluminescence was significantly increased 20 days after the start of the challenge with a return to control values after 27 days.

Ceruloplasmin activity, which is linked to the inflammatory response, tended to be reduced one month after the parasitical challenge in fish fed the control diet. This reduction of inflammation caused by parasitic infection was also suggested by authors who showed a reduction of neutrophil and oesinophil counts 10-27 days and 27 days after the start of the challenge, respectively (Alvarez-Pellitero et al. 2008). Similarly, the tendency for reduction of anti-protease activity observed in the present study after four weeks of a parasitical challenge was also observed by previous authors (Muñoz et al. 2007). However, these authors showed that this reduction after four weeks followed a transient increase 12 and 19 days after the start of the challenge. Lysozyme activity was low (80 units/ml) and unaffected by the challenge in the present study. A previous study failed to detect any lysozyme activity in sharpsnout seabream either before or after E. leei challenge (Golomazou et al. 2006).

The tendency for decreased values of anti-protease (linked to the anti-parasitical and/or anti-bacterial arsenal of the fish), anti-bacterial activities (lysozyme and complement) and inflammatory activity (ceruloplasmin) of the challenged fish observed in the present study suggested that the parasite suppressed the immune and inflammatory responses of the fish through a successful and unknown evasion mechanism efficient four weeks after the start of the cohabitation with parasitised fish. Despite this, the re- 
duction of immunological parameters observed in control fish tended to be less critical when fish were fed the functional supplement for one month before the challenge.

The use of a functional feed additive at the inclusion level used in this trial, as recommended by the manufacturer $(0.3 \%$ in the diet), for four weeks prior to challenge did not significantly improve growth or survival of infected fish. Previous studies in gilthead seabream indicated some efficacy of this natural supplement against enteromyxosis, expressed through the reduction of the prevalence and intensity of infection and improvement of the condition of fish in an experimental challenge with this parasite, although it did not totally clear off the infection (Palenzuela et al. 2017). Such effect was not obvious for sharpsnout seabream, where the high prevalence and mean intensity of infection reached in recipient fish at the end of the trial further demonstrates its extreme susceptibility to E. leei.

Differences between functional and control diets would probably be more pronounced in a less acute infection model with lower parasite intensity and prevalence. Profound differences between gilthead seabream and sharpsnout seabream in the immune mechanisms involved in the response to $E$. leei have been shown in previous studies (Muñoz et al. 2007, Alvarez-Pellitero et al. 2008). In gilthead seabream, enteromyxosis courses as a chronic disease and the main clinical effects are related to suppressed growth and cachexia. However, it rarely evolves to a mass mortality outbreak unless other triggers such as water reuse, high temperatures and/or secondary infections occur (reviewed in Sitjà-Bobadilla and Palenzuela 2011).

Recent studies have shown that dietary supplements like butyrate can alleviate the outcome of experimentally induced enteromyxosis in gilthead seabream by partially preventing anorexia, growth suppression and the associated clinical signs, although it did not decrease infection levels (Piazzon et al. 2017). Taking this into account, different dietary levels, mode and duration of administration of functional ingredients should also be explored to improve their efficacy.

In conclusion, this study demonstrated an efficient host-parasite model for experimental infection of sharpsnout seabream with $E$. leei, which reproduced the rapid and effective progression of the disease observed in the field. Given the high pathogenicity of E. leei in sharpsnout seabream, it is not surprising that nutraceutical strategies could not prevent clinical enteromyxosis in this trial, in which a very high infection pressure was applied. However, the drop observed in some immune-related parameters in challenged control fish appeared reduced in medicated fish, indicating a biological effect of the supplement that may become effective if functional feed was supplied earlier before the rise of water temperatures. Further attempts to find effective prophylactic and therapeutic strategies against enteromyxosis are paramount for the farming rebooting of sharpsnout seabream and should involve the administration of functional feeds at different periods, administration levels and duration.

Acknowledgments. This work was made possible through a H2020-SFS-2014-2015 project entitled: ParaFishControl: Advanced Tools and Research Strategies for Parasite Control in European farmed fish (grant agreement no: 634429). This publication reflects only the authors' view, and the European Commission cannot be held responsible for any use which may be made of the information contained herein.

\section{REFERENCES}

Alvarez-Pellitero P., Palenzuela O., Sitjà-Bobadilla A. 2008: Histopathology and cellular response in Enteromyxum leei (Myxozoa) infections of Diplodus puntazzo (Teleostei). Parasitol. Int. 57: 110-120.

Athanasopoulou F., Prapas T., Rodger H. 2001: Diseases of Puntazzo puntazzo Cuvier in marine aquaculture systems in Greece. J. Fish Dis. 22: 215-218.

Coutteau P. 2016: Functional feed additives to prevent disease in farmed shrimp. Aquafeed 7: 24-27.

Cuadrado M. 2010: Enteromyxosis produced by Enteromyxum leei (Diamant, Lom and Dyková 1994) in Mediterranean sparids of commercial interest. Ph.D Thesis, Autonomous University of Barcelona, Barcelona, 180 pp. (In Spanish.)

Defoirdt T., Sorgeloos P., Bossier P. 2011: Alternatives to antibiotics for the control of bacterial disease in aquaculture. Curr. Opin. Microbiol. 14: 251-258.

Diamant A. 1997: Fish-to-fish transmission of a marine myxosporean. Dis. Aquat. Org. 30: 99-105.

Diamant A., Lom J., Dyková I. 1994: Myxidium leei n. sp., a pathogenic myxosporean of cultured sea bream Sparus aurata. Dis. Aquat. Org. 20: 137-141.

Favaloro E., Lopiano L., Mazzola A. 2002: Rearing of sharpsnout seabream (Diplodus puntazzo, Cetti 1777) in a Mediterranean fish farm: monoculture versus polyculture. Aquac. Res. 33: 137-140.
Favaloro E., Mazzola A. 2003: Shape change during the growth of sharpsnout seabream reared under different conditions in a fish farm of the southern Tyrrhenian Sea. Aquac. Eng. 29: 57-63.

Golomazou E., Athanassopoulou F., Karagouni E., TsagoZis P., Tsantilas H., Vagianou S. 2006: Experimental transmission of Enteromyxum leei Diamant, Lom and Dyková, 1994 in sharpsnout sea bream, Diplodus puntazzo C. and the effect on some innate immune parameters. Aquaculture 260: 44-53.

Henry M., Fountoulaki E. 2014: Optimal dietary protein/lipid ratio for improved immune status of a newly cultivated Mediterranean fish species, the shi drum Umbrina cirrosa, L. Fish Shellfish Immunol. 37: 215-219.

Henry M., Nikoloudaki C., Tsigenopoulos C., Rigos G. 2015: Strong effect of long-term Sparicotyle chrysophrii infection on the cellular and innate immune responses of gilthead sea bream, Sparus aurata. Dev. Comp. Immunol. 51: 185-193.

Henry M.A., Alexis M.N., Fountoulaki E., Nengas I., Rigos G. 2009: Effects of a natural parasitical infection (Lernanthropus kroyeri) on the immune system of European sea bass, Dicentrarchus labrax L. Parasite Immunol. 31: 729-740.

Hernández M.D., Martinez F.J., Garcia B.G. 2008: Diplodus puntazzo, a new species in aquaculture: biology and culture. In: S.H. Schwartz (Ed.), Aquaculture Research Trends. Nova Science Publishers Inc., New York, pp. 237-261. 
Kokou F., Rigos G., Henry M., Kentouri M., Alexis M. 2012: Growth performance, feed utilization and non-specific immune response of gilthead sea bream (Sparus aurata L.) fed graded levels of a bioprocessed soybean meal. Aquaculture 364-365: $74-81$.

Le Breton A., Marques A. 1995: Occurrence of an histozoic Myxidium infection in two marine cultured species: Puntazzo puntazzo and Pagrus major. Bull. Eur. Assoc. Fish Pathol. 15: 210-212.

Muñoz P., Cuesta A., Athanassopoulou F., Golomazou H., Crespo S., Padrós F., Sitjà-Bobadilla A., Albiñana G., Esteban M.A., Alvarez-Pellitero P., Meseguer J. 2007: Sharpsnout sea bream (Diplodus puntazzo) humoral immune response against the parasite Enteromyxum leei (Myxozoa). Fish Shellfish Immunol. 23: 636-645.

Palenzuela O. 2006: Myxozoan infections in Mediterranean mariculture. Parassitologia 48: 27-29.

Palenzuela O., Del Pozo R., Piazzon M.C., Isern-Subich M.M., Ceulemans S., Coutteau P., Sitjà-Bobadilla A. 2017: Functional feed additives can reduce the impact of an Enteromyxum leei infection on performance and disease severity: evidence from an experimental challenge with gilthead sea bream. Int. Aquafeed 20: 14-19.

Piazzon M.C., Calduch-Giner J.A., Fouz B., Estensoro I., Simó-Mirabet P., Puyalto M., Karalazos V., Palenzuela O., Sitjà-Bobadilla A., Pérez-Sánchez J. 2017: Under control: how a dietary additive can restore the gut microbiome and proteomic profile, and improve disease resilience in a marine teleostean fish fed vegetable diets. Microbiome 5: 164.
Picard-Sánchez A., Estensoro I., del Pozo R., Piazzon M.C., Palenzuela O., Sitjà-Bobadilla A. 2019: Acquired protective immune response in a fish-myxozoan model encompasses specific antibodies and inflammation resolution. Fish Shellfish Immunol. 90: 349-362.

Rigos G., Christophilogiannis P., Yiagnisi M., Andriopoulou A., Koutsodimou M., Nengas I., Alexis M. 1999: Myxosporean infections in Greek mariculture. Aquac. Int. 7: 361-364.

Rigos G., Samartzis A., Henry M., Fountoulaki E., Cotou E., Sweetman J., Davies S., Nengas I. 2010: Effects of additive iron on growth, tissue distribution, haematology and immunology of gilthead sea bream, Sparus aurata. Aquac. Int. 18: 1093-1104.

Sánchez-García N., Raga J.A., Montero F.E. 2014: Risk assessment for parasites in cultures of Diplodus puntazzo (Sparidae) in the Western Mediterranean: prospects of cross infection with Sparus aurata. Vet. Parasitol. 204: 120-133.

Sitjà-Bobadilla A., Calduch-Giner J., Saera-Vila A., Palenzuela O., Alvarez-Pellitero P., Pérez-Sánchez J. 2008: Chronic exposure to the parasite Enteromyxum leei (Myxozoa: Myxosporea) modulates the immune response and the expression of growth, redox and immune relevant genes in gilthead sea bream, Sparus aurata L. Fish Shellfish Immunol. 24: 610-619.

Sitjà-Bobadilla A., Palenzuela O. 2011: Enteromyxum species. In: P.T.K. Woo and K. Buchmann (Eds.), Fish Parasites: Pathobiology and Protection. CABI, Wallingford, pp. 163-176.

Cite this article as: Henry M.A., Kokou F., Palenzuela O., Pyrenis G., Rigos G. 2020: Experimental infection model with the bivalvulid Enteromyxum leei (Myxidiidae) in the sharpsnout seabream, Diplodus puntazzo (Sparidae), and evaluation of the antiparasitic efficacy of a functional diet. Folia Parasitol. 67: 024. 